\title{
DETC2018-85452
}

\section{TOWARDS THE DESIGN OF RESILIENT WASTE-TO-ENERGY SYSTEMS USING BAYESIAN NETWORKS}

\author{
W. H. Jonathan Mak \\ University of Cambridge \\ Cambridge, UK \\ Liu Ziqi \\ National University of Singapore \\ Singapore
}

\author{
Michel-Alexandre Cardin \\ National University of Singapore \\ Singapore \\ P. John Clarkson \\ University of Cambridge \\ Cambridge, UK
}

\begin{abstract}
The concept of resilience has emerged from various domains to address how systems, people and organizations can handle uncertainty. This paper presents a method to improve the resilience of an engineering system by maximizing the system economic lifecycle value, as measured by Net Present Value, under uncertainty. The method is applied to a Waste-to-Energy system based in Singapore and the impact of combining robust and flexible design strategies to improve resilience are discussed. Robust strategies involve optimizing the initial capacity of the system while Bayesian Networks are implemented to choose the flexible expansion strategy that should be deployed given the current observations of demand uncertainties. The Bayesian Network shows promise and should be considered further where decisions are more complex. Resilience is further assessed by varying the volatility of the stochastic demand in the simulation. Increasing volatility generally made the system perform worse since not all demand could be converted to revenue due to capacity constraints. Flexibility shows increased value compared to a fixed design. However, when the system is allowed to upgrade too often, the costs of implementation negates the revenue increase. The better design is to have a high initial capacity, such that there is less restriction on the demand with two or three expansions.
\end{abstract}

Keywords: Resilience, Bayesian Networks, infrastructure systems, complex systems design

\section{INTRODUCTION}

Infrastructure systems such as telecommunications, power, waste disposal and transport networks form the backbone of most societies. Failure in these services can bring major disruption to a community and recovery can incur substantial time and cost. Furthermore, infrastructure systems characteristically have relatively long life cycles, typically more than 10 years, leading to a range of uncertainties and involving major investments. How these engineered systems are designed to accommodate this uncertainty is therefore paramount to ensure the success of such projects.

The concept of "resilience" has emerged in literature and has been found to address these concepts in a number of fields. The term "resilience" was first popularized by Holling [1] within the field of ecology to assess the stability and resilience of interacting populations and the environment. In their work, the term is defined as the "persistence of relationships within a system and is a measure of the ability of these systems to absorb changes of state variables, driving variables, and parameters, and still persist". This concept of a system's interaction with the environment and surviving disturbances is similar to the foundations for resilience in many other fields including supply chain management [2], crisis management [3], psychology [4] and resilience engineering [5]. Thus, resilience has traditionally been associated with negative connotations: the ability to recover from adversity or trauma. However, there is now growing recognition, especially in management literature, that resilience not only allows for recovery from disruption, but also to allows for the ability to thrive and prosper despite difficult times [6]. 
While there has been substantial literature describing the concept of resilience, there is comparatively less work on quantitatively assessing resilience. Here, resilience is taken to be the design of the system that maximizes the system's lifecycle value, as measured by Net Present Value (NPV), under seen and unforeseen uncertainties. This paper presents the evaluation of a Waste-to-Energy (WTE) system in Singapore and the impact of robust and flexible design strategies on the NPV of the system. The effect of robustness is explored by optimizing the initial capacity of the system while flexibility allows the system to be upgraded through expansion. Bayesian Networks (BNs) are introduced to select the type of expansion, whether centralized or decentralized, as well as the sites for expansion. The resilience of the system is evaluated by varying the volatility of the demand on the system in order to assess the impact on the NPV and understand the merits of different infrastructure designs

The remainder of this paper is structured as follows: Related work in resilience and BNs are presented in Section 2. The methodology of this work is shown in Section 3 and applied to the WTE system in Singapore in Section 4. The impact of varying volatility on NPV and therefore resilience is presented in Section 5.

\section{BACKGROUND}

\subsection{Resilience in Engineering Systems}

Studies in a number of disciplines have emphasized the need for resilience to overcome disturbances and thrive despite difficult times. With respect to designing engineering systems, a recent literature study [7] spanning the fields of engineering, organizational/management, and ecological works has summarized three main characteristics for further consideration: absorbing disturbances, adapting for change, and thriving for the future. This was mapped to the corresponding engineering design ilities of robustness, adaptability and flexibility for the design of resilient engineering systems. It should be noted, however, that there is significant overlap in the definitions of adaptability and flexibility in literature. Here, these subtleties are briefly defined to give requirements for design of resilient engineering systems.

\subsubsection{Robustness}

The property of robustness stems from engineering literature and may be defined as the ability to be "insensitive towards changing environments" [8]. With robustness, the system is able to maintain the required performance, despite disturbances, through some redundancy or tolerance designed into the system [9]. In an infrastructure system, for example, bridges are designed to be robust so that it withstands extra loading from increased traffic or fluctuations in wind speed/direction. Robust designs may be more cost efficient when the disturbances are predictable, but may fail if there are substantial, unexpected influences on the system which push the system outside the design margins. As such, robustness may be better suited for systems where the uncertainties are more understood or where the demands of the system are unlikely to change throughout the system lifecycle. However, infrastructure systems in particular are usually complex and system lifecycles tend to span over 10 years making uncertainty difficult to predict. Therefore, while the system still has to be robust, it is not a sufficient condition if the system is to be resilient and perform for significant periods where there may be unknowns.

\subsubsection{Adaptability}

Over the lifecycle of a system, there may be instances where the system has to change to accommodate influences on the system. The margins of the robust design may be exceeded and therefore the system has to change to maintain satisfactory performance. This may be considered through the properties of adaptability and flexibility. There is, however, a lack of consensus concerning these definitions in literature and these two terms are often used synonymously. Here, adaptability is used to denote where the system can change through an internal change agent $[8,10]$. An internal change agent is where change is instigated within the system automatically without the need for external action and serves to move the system to a predefined performance level. This could be in the form of internal control systems and feedback loops where the system changes automatically to maintain system performance. For telecommunications infrastructure, this could be the automatic rerouting of network traffic based on the current demand. Since the changes occur automatically in the system, these changes must be planned and anticipated during the conceptual design stage so that the system continues to operate within the required boundaries. Indeed, some unforeseen event could still push the system outside these initial design boundaries which cannot be automatically rectified leading to failure. As such, a system upgrade, or flexible design discussed in section 2.1.3, may be more appropriate. An adaptable design is therefore useful where it is impractical to make the system excessively robust through large redundancies and instead allows the system to change automatically. This requires some foresight into the environment in which the system is deployed and therefore may be useful, as similarly for robust designs, where uncertainties are relatively more understood in the near future or where the demands on the system is unlikely to change throughout the lifecycle.

\subsubsection{Flexibility}

In the event a substantial change or upgrade is required for the system, a flexible design may be adopted. This allows the system to change for new opportunities or accommodate disturbances which the original system was not designed for. Flexibility therefore contrasts with robustness and adaptability in that it does not serve to maintain normal operations, but instead, it allows the system to change its performance boundaries. The concept of flexibility and adaptability may be distinguished by the location of the change agent. In the adaptable case, the change agent is located within the system leading to automatic change. On the other hand, the flexible system has the change agent external to the system and allows a decision maker to 
change the requirements of the system [10]. A flexible system may therefore be designed so that it has a number of options for the decision maker and flexibility makes it easy to change or upgrade these options. This can be achieved through modularity, platform design or interface design [8]. The Ponte 25 de Abril suspension bridge over the Tagus River demonstrates flexible design in that it was built with the strength to accommodate a secondary railroad deck when demand was sufficient [11]. In essence, the bridge was designed so that it could be upgraded easily when needed. Flexibility is therefore important where the system requirements could change over their lifecycles, such as for infrastructure systems, and uncertainties are relatively harder to predict making it a necessary condition for resilience.

\subsection{Requirements for Resilient Engineering Infrastructure Systems}

The three properties for resilient engineering systems were briefly described in the previous sections. However, for some infrastructure systems, it may be impractical to keep physically switching between components as per the adaptable strategy. That is for road networks, power plants and, in this case, waste disposal systems, it can be difficult to change assets once installed. For infrastructure projects, the properties of robustness and flexibility are therefore more important. Essentially, the initial robustness or capacity of the system should be carefully designed and there should be due diligence in assessing how to upgrade the system through flexibility. By designing for robustness and flexibility, this ensures that the system can cope with immediate day-to-day pressures as well as giving options to accommodate for future uncertainties. Further design strategies for resilience therefore focuses on robustness and flexibility.

Having defined the conceptual requirements for resilient engineering infrastructure systems in the previous section, existing models for quantitatively evaluating resilience is now discussed. For example, system dynamic models are used for modeling supply chains [2], mathematical models used for network analysis [12] and petrochemical supply chains [13]. BNs have been applied to inland water ports [14] and genetic algorithms have been used to model infrastructure restoration [15]. A more extensive review can be found from Hosseini et al [16]. In such cases, resilience is measured by the time or ability it takes for the system to return to normal or recover following some disturbance. Here, it is assumed that by designing robust systems, the system response is adequate to return to normal operations. There has further been growing recognition that systems do not just undergo disturbance in the negative sense, but also disruptive opportunities may be presented in the future. As such, resilience should also incorporate the idea of being able to "survive and thrive" and is addressed through flexible strategies which allows the system to operate with new requirements.

In this paper, the WTE system lifecycle value is modeled through NPV analysis such that designing a resilient system involves optimizing the robustness and flexibility of the system to maximize the NPV over a range of uncertainties. To the authors' knowledge there has not been resilience analysis that incorporates the selection of flexible strategies through BNs such that both robustness and flexibility is considered in the design of resilient systems. Furthermore, this paper explores the impact of robustness and flexibility on the system by varying the uncertainty over time.

\section{METHODOLODY}

This work follows a five step framework guided by Cardin's flexibility cycle [17] and builds upon work by Liu [18]. The framework comprises Baseline Design, Uncertainty Recognition, Concept Generation, Design Space Exploration and Resilience Analysis and are discussed generally in this section before being applied to the WTE system. The relationship between the five steps are shown in Figure 1.

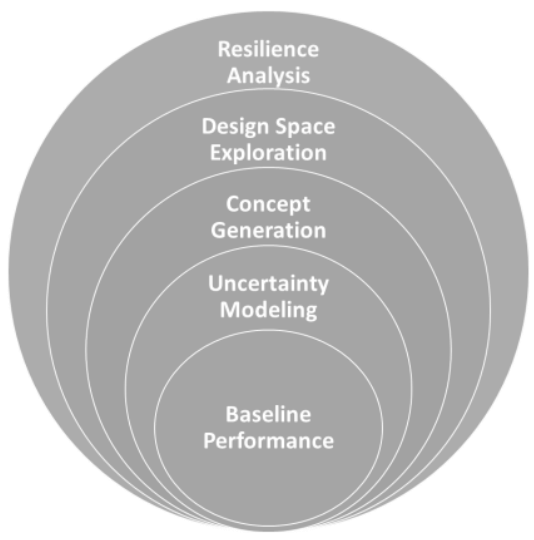

\section{FIGURE 1 - RELATIONSHIP OF FIVE STEP FLEXIBILITY} FRAMEWORK

\subsection{Baseline Performance}

This first step assesses the NPV of the system with no flexibility upgrade options to establish a benchmark for comparing to other infrastructure designs generated in latter steps. NPV models are developed under deterministic conditions based on deterministic forecasts for uncertainty factors. The initial robustness of the system can be optimized given these forecasts but there are no flexibility options.

\subsection{Uncertainty Modeling}

The system is subject to uncertainties which may affect future performance. This step identifies these sources of uncertainty through experts' and designers' experience which can be then modeled by collecting historic data and statistical analysis. For this study, the uncertainty will be modelled through Geometric Brownian Motion to simulate demand on the system.

\subsection{Concept Generation}

In order for a system to be resilient, the system may be designed to be robust, but may also have to change if the original demands are exceeded. In such a case, flexible strategies should 
be considered which can include expanding capacity, deferring investment or switching product lines. This step involves understanding the flexible options available to the system through real options analysis [19] and a number of techniques have been developed to systematically generate these concepts [20, 21]. In particular, a BN approach has been used to study the high risk components in a system that should be considered for flexible strategies [22]. Once these strategies are conceptualized, decision rules can be put in place to embed flexibility into the design.

This paper builds on this work by extending the use of BNs for decision rules. That is, once the potential strategies are identified, the $\mathrm{BN}$ is used to determine when to execute the strategy given the uncertainties observed on the system. Cardin \& Hu [23] and Liu [18] have performed a flexibility study on WTE systems using IF statements as decision rules. By incorporating BNs into this the decision rules, a broader scope of uncertainties can be accounted for when deciding whether to pursue the flexible option compared to IF statements. Furthermore, BNs can include qualitative as well as quantitative data as well as giving the decision-maker an intuitive visualization of the system's dependencies.

\subsection{Design Space Exploration}

The fourth step involves evaluating the Expected Net Present value (ENPV) of the system with the embedded flexibility. The search space for the system is generated through Monte Carlo simulation from Step 2 to give a distribution of performance outcomes for comparison. The optimal robustness can be investigated through simulated annealing to find the optimal initial capacity for the design.

The different design options can then be compared with the Baseline Design in Step 1 where there is no flexibility. This allows the designers to discern whether the flexible strategy is worth the additional investment cost. The difference between the ENPV of the flexible design and the NPV of the fixed design in Step 1 is the value of flexibility.

\subsection{Resilience Analysis}

As discussed in Section 2, a resilient system should be both robust and flexible in order to operate in a range of uncertainties. For example, when upgrading system capacity, consideration should be given to how much to increase capacity each time and the system's initial robustness boundaries should also be assessed. If the initial robust margins are sufficiently large, this may allow the investment of the flexible strategy in the future to be deferred in order to save on upgrade costs. On the other hand, if it is anticipated that requirements can often change, it may be better to save on initial investments and spend when there is necessary change.

As such, resilience analysis examines the effect of changing the system uncertainties on the robust and flexibility requirements. This is achieved by varying volatility in the stochastic demand to evaluate impact on the ENPV. Through the results of these simulations, the system's robustness and flexibility can then be optimized in order to design a resilient system which can accommodate uncertainties in the short and long term. The pipeline of the model can therefore be visualized as in Figure 2.

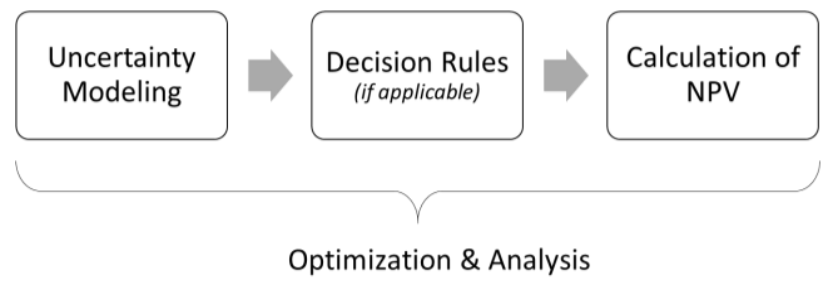

FIGURE 2 - PIPELINE OF MODEL

\section{APPLICATION TO WASTE TO ENERGY SYSTEM}

The framework discussed in the previous section is now applied to a WTE system in Singapore building on work by Liu [18] and Cardin \& Hu [23]. Singapore is divided into six sectors by public waste collector contractors which collect waste within each sector before transporting to the centralized processing plant. An alternative option exists for six smaller anaerobic digestion $(\mathrm{AD})$ processing plants to be developed in each of the six sectors in a decentralized manner. In such a case, instead of transporting the waste to a main central site, the waste can be processed in each of the six sectors. This decentralized system could be a potential solution to combat increasing waste generation and providing a more sustainable processing method.

The objective of this paper is to therefore assess the merits of moving to a decentralized system and evaluate how to design the system so that it is resilient to short term and long term fluctuations in demand. Following the framework, the baseline design deterministically models the centralized and decentralized system with no increases in capacity. In the uncertainty recognition step, the demand on the system is simulated for each sector through 15 year time periods. Based on the demand, the sectors for capacity expansion and decentralization are selected through the $\mathrm{BN}$ representation of the decision rules. The ENPV is then calculated and the volatility is varied to understand the impact of different design strategies.

\subsection{Step 1: Baseline Performance}

There are two main design concepts for the WTE system: centralized and decentralized development. Both of these are simulated with deterministic demands in the first instance with no increases in capacity to give a benchmark NPV for comparison in later stages. Further details, coefficient and data on the model can be obtained from [18, 23].

\subsubsection{Model Development of Centralized Design}

To calculate the NPV of the centralized WTE system, the model is constructed by considering a planning horizon of $T=$ 15 time periods where demand is assumed to be known. The total capacity installed is denoted by $x$, and $d^{t}$ is the total capacity demand at time, $t$. The NPV may therefore be 
maximized over the planning horizon by finding the capacity $x$, which fulfills demand, $d$ as follows

$$
\begin{gathered}
\operatorname{Max} N P V=-C^{0}(x)+\sum_{t=1}^{T}\left(\frac{1}{1+\lambda}\right)^{t}\left[R^{t}\left(x, d^{t}\right)-C^{t}\left(x, d^{t}\right)\right] \\
\text { s.t } 0 \leq x \leq x_{\max } \\
d^{t} \geq 0, \forall t
\end{gathered}
$$

$C^{0}(x)$ is the initial cost of investing into the plant, $\lambda$ is the discount rate, $R^{t}\left(x, d^{t}\right)$ is the revenue of the system, and $C^{t}\left(x, d^{t}\right)$ is the cost function in time.

The revenue of the WTE systems at year $t$ consists of the selling revenue from refuse derived fuel $\left(R_{R D F}^{t}\right)$, metal $\left(R_{M}^{t}\right)$, biogas $\left(R_{B}^{t}\right)$, and water $\left(R_{W}^{t}\right)$ as well as a tipping fee for collecting solid waste $\left(R_{\text {Tip }}^{t}\right)$. This is given by the equation

$$
R^{t}=R_{R D F}^{t}+R_{M}^{t}+R_{B}^{t}+R_{W}^{t}+R_{\text {Tip }}^{t}
$$

These are assumed to be proportional to the amount of waste treated in the plant. The costs involved with the centralized design include: transportation $\operatorname{cost}\left(C_{T S}^{t}\right)$, disposal $\operatorname{cost}\left(C_{D}^{t}\right)$, land rental $\operatorname{cost}\left(C_{L D}^{t}\right)$, operation and maintenance $\operatorname{cost}\left(C_{O M}^{t}\right)$, resource consumption cost $\left(C_{R C}^{t}\right)$ and pollution $\operatorname{cost}\left(C_{P L}^{t}\right)$. This is summarized in the below equation

$$
C^{t}=C_{T S}^{t}+C_{D}^{t}+C_{L D}^{t}+C_{O M}^{t}+C_{R C}^{t}+C_{P L}^{t}
$$

The transportation cost results from the fuel consumption for collecting the waste in each sector and transporting it to the central facility. There is a cost of disposal, $C_{D}^{t}$, incurred when there is unmet demand in the WTE plant and the untreated waste needs to be disposed at incineration plants or landfills. The land rental cost is proportional to the land needed for installed capacity, while the operational and maintenance cost is assumed to be proportional by some coefficient, $\pi$, to the initial cost. The WTE system consumes energy including electricity and natural gas and is assumed to be proportional to the amount of solid waste treated. The cost of pollution results from the cost of treating the $\mathrm{CO}_{2}$ emissions from the WTE system.

\subsubsection{Model Development of Decentralized Design}

The calculations for the decentralized design are similar but instead of having all the waste transported to one central site, the waste is transported to six smaller scale plants in each of the six sectors. The total demand, $d^{t}$, is assumed to be distributed among the six sectors according to population density. The total capacity, $x$, is therefore the sum of the capacity in each sector. The total revenue is updated accordingly by summing over the six sectors.

The NPV model for the decentralized design is therefore

$$
\operatorname{Max} N P V=-\sum_{i=1}^{6} C^{0}\left(x_{i}\right)+\sum_{t=1}^{T} \sum_{i=1}^{6}\left(\frac{1}{1+\lambda}\right)^{t}\left(R^{t}-C^{t}\right)
$$

With the models for the centralized and decentralized models defined, the NPVs for both designs are calculated for a 15 year horizon with the demand deterministically projected based historical data from the National Environmental Agency (NEA) annual report [24]. For the centralized model, given growth rate $\mu$, the waste is given by the equation

$$
S^{t}=S^{t-1}(1+\mu)
$$

where $S^{t}$ is the waste generated at year $t$. For the decentralized case, the amount of waste in each sector is estimated from the population density in each sector, $i$ from

$$
S_{i}^{t}=p_{d} * S^{t}
$$

To make the design capacity more practical, the capacity is assumed to be in multiples of 50 tonnes per day (tpd). A

\begin{tabular}{|c|c|c|c|c|c|c|c|}
\hline NPV & \multicolumn{6}{|c|}{ Initial Capacity } & \multirow{2}{*}{$\begin{array}{c}\text { Total } \\
\text { Capacity } \\
\text { (tpd) }\end{array}$} \\
\hline $\begin{array}{c}(\mathrm{S} \$ \\
\text { Million) }\end{array}$ & $\begin{array}{c}\text { Sector } \\
1\end{array}$ & $\begin{array}{c}\text { Sector } \\
2\end{array}$ & $\begin{array}{c}\text { Sector } \\
3\end{array}$ & $\begin{array}{c}\text { Sector } \\
4\end{array}$ & $\begin{array}{c}\text { Sector } \\
5\end{array}$ & $\begin{array}{c}\text { Sector } \\
6\end{array}$ & \\
\hline 251 & 1450 & 900 & 850 & 800 & 550 & 500 & 5050 \\
\hline
\end{tabular}
simulated annealing optimization was conducted to find the optimal configuration of initial capacity in each sectors to maximize the NPV. For the centralized design, an initial capacity of 5200 tpd gave a NPV of $\mathbf{\$} \$ 243$ million. The initial capacities and NPV for the decentralized design is summarized in Table 1.

TABLE 1 - NPV OF FIXED DECENTRALIZED DESIGN UNDER DETERMINISTIC ANALYSIS

For these results, the decentralized design is shown to surpass the centralized design in terms of NPV. This is due to the savings in the transportation cost in the decentralized design.

\subsection{Step 2: Uncertainty Modeling}

The previous step used a projection for demand on the system based on a fixed growth rate. In reality, this is seldom the case and uncertainty is now introduced into the model in this step by modeling waste generation in each site with Geometric Brownian Motion. This is formulated in the following equation

$$
d S_{i}^{t}=\mu S_{i}^{t} d t+\sigma_{i} S_{i}^{t} d W_{t}
$$

where $S_{i}^{t}$ is the waste collected in sector $i, \mu$ denotes the trend or growth rate, $\sigma_{i}$ is the volatility and $W_{t}$ is the Wiener process. The growth rate and volatility are estimated to be $1.71 \%$ and $2.03 \%$ respectively based on historical data. A Monte Carlo Simulation is ran 2000 times and the ENPV for the centralized model can be obtained from 


$$
\operatorname{Max} E N P V=\sum_{l}^{L} p_{l}\left\{-C^{0}(x)+\sum_{t=1}^{T}\left(\frac{1}{1+\lambda}\right)^{t}\left(R_{l}^{t}-C_{l}^{t}\right)\right\}
$$

where $p_{l}$ is the probability associated with scenario $l$ and $L$ is the total number of simulations ran. The decentralized model is similarly

$$
\begin{aligned}
\operatorname{Max} E N P V=\sum_{l}^{L} & p_{l}\left\{-\sum_{i=1}^{6} C^{0}\left(x_{i}\right)\right. \\
& \left.+\sum_{t=1}^{T} \sum_{i=1}^{6}\left(\frac{1}{1+\lambda}\right)^{t}\left(R_{l}^{t}-C_{l}^{t}\right)\right\}
\end{aligned}
$$

Running the simulations with uncertain waste generation, the optimal initial capacity for the centralized design is 5200 tpd giving an ENPV of $\mathbf{S} \$ 242$ million. The decentralized case is summarized in Table 2.

\begin{tabular}{|c|c|c|c|c|c|c|c|}
\hline \multirow{2}{*}{$\begin{array}{l}\text { ENPV } \\
\text { (S\$ } \\
\text { Million) }\end{array}$} & \multicolumn{6}{|c|}{ Initial Capacity } & \multirow{2}{*}{$\begin{array}{c}\text { Total } \\
\text { Capacity } \\
\text { (tpd) }\end{array}$} \\
\hline & Sector & Sector & Sector & $\begin{array}{c}\text { Sector } \\
4\end{array}$ & $\begin{array}{c}\text { Sector } \\
5\end{array}$ & $\begin{array}{c}\text { Sector } \\
6\end{array}$ & \\
\hline 250 & 1600 & 900 & 850 & 800 & 550 & 500 & 5200 \\
\hline
\end{tabular}

\section{TABLE 2 - ENPV OF FIXED DECENTRALIZED DESIGN} UNDER UNCERTAINTY

The ENPV for both cases are, as expected, less than the NVP as calculated in the previous step due to the uncertainty added into the system. Again, the decentralized case performs better than the centralized case.

\subsection{Step 3: Concept Generation \& Selection}

The previous two steps incorporated an optimization to find the initial robust margins for initial capacity. This steps incorporates flexibility into the analysis in order to understand how the system can change for future needs. The flexible strategy to expand the capacity of the WTE plants after installation is simulated since the demand fluctuation is the major uncertainty. This allows for the WTE plant to expand modularly given an increase in demand, but also mitigates risk in having too large a site if the forecast demand is not met.

Decision rules are established to determine when to enable the flexible strategies. The decision rules for expanding capacity in the decentralized case is therefore: (1) Determine if there is unmet capacity and whether the total capacity is less than the maximum capacity after expansion. (2) Determine whether the main site or non-main sites should be expanded. (3) Determine which of the six sectors should be expanded if in a decentralized manner. This is summarized in Figure 3.

Here, BNs are presented to capture these decision rules. BNs are chosen in particular for the ability to capture a range of uncertainties, both qualitative and quantitative, the power of using network inference for causal reasoning as well as providing an intuitive interface for the decision maker to visualize interdependencies.

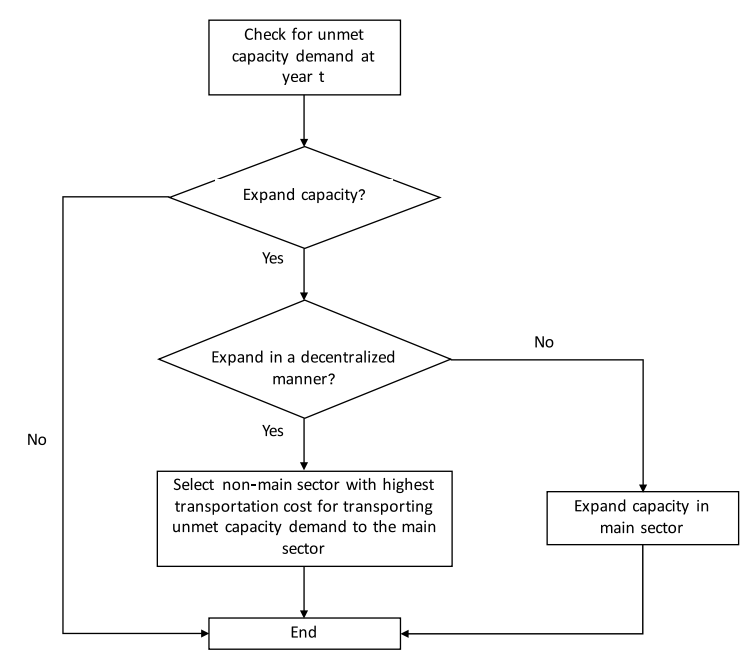

\section{FIGURE 3 - FLOW CHART OF DECISION-MAKING PROCEDURES FOR DECENTRALIZED DESIGN}

BNs are directed acyclic graphical models which are used to represent a set of variables and their interdependencies. The variables are shown as nodes and the interdependencies, input via conditional probability tables, are represented as edges in the graph. Observed variables, say whether there is unmet demand in year $t$, can be input into the network and the probabilities of the other variables can be updated through inference in the network. Mathematically, inference is applied using Bayes' Rule as below. Further information on mathematical details and implementation can be obtained from Pearl or Nielson \& Jensen $[25,26]$.

$$
P(A \mid B)=\frac{P(B \mid A) P(A)}{P(B)}
$$

The $\mathrm{BN}$ in this study is setup to assess whether the decentralized design needs to be upgraded and, in the case that an expansion is necessary, the sector that should be upgraded is indicated. Figure 4 shows a screenshot from BN software, Netica [27], for illustration of the BN.

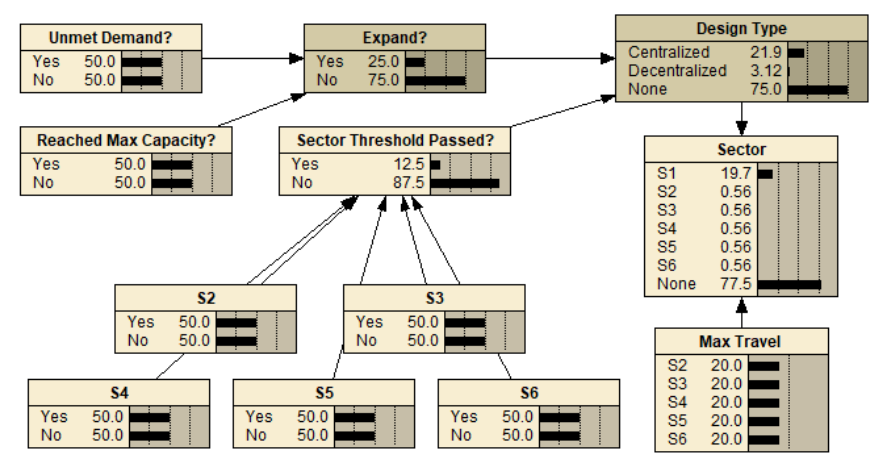

FIGURE 4 - BAYESIAN NETWORK DECISION RULES

Each box, or node represents a variable and the black bars show the prior and posterior probabilities of the states. The Boolean decisions, whether to expand or not etc., are represented as yes 
or no states in the nodes. The nodes S2 - S3 holds information on whether the non-main sector capacity thresholds have been breached. If all the non-main sector thresholds are exceeded, the "Sector Threshold Passed?" node updates to yes. The "Design Type" node indicates whether this should be centralized or decentralized expansion, if any, and depends on observations given in the nodes "Expand?" and "Sector Threshold Passed?". The "Sector" node then classifies which sector should be expanded, if any, with $\mathrm{S} 1$ representing the main sector and S2S6 being the non-main sectors. The "Max Travel" node indicates the sector which incurs the maximum travel cost. The conditional probability tables for each node were based on the logic in Figure 3.

One major advantage of network inference from an uncertainty point of view, is that not all variables have to be observed for the probabilities to be updated. This allows a decision maker to understand the impact of what-if scenarios on the system with limited information and decide whether the system should be changed or in this case, expanded. For example, the following figure shows the BN with observations in the greyed-out nodes: "Expand?" = yes, "Sector Threshold Passed?" = yes and "Max Travel" = S5. The rest of the network updates through inference and indicates that the expansion must be decentralized. This is due to the observation that the sector capacity thresholds have been passed. Had the thresholds not have been exceeded, centralized expansion would have been recommended. The sector to be expanded, as shown in the "Sector" node is S5, which follows from the "Max Travel" observation which indicates the sector with the highest travel cost to offset unmet demand. The network also indicates that all sectors, nodes "S2:S6" are likely to have exceeded the threshold.

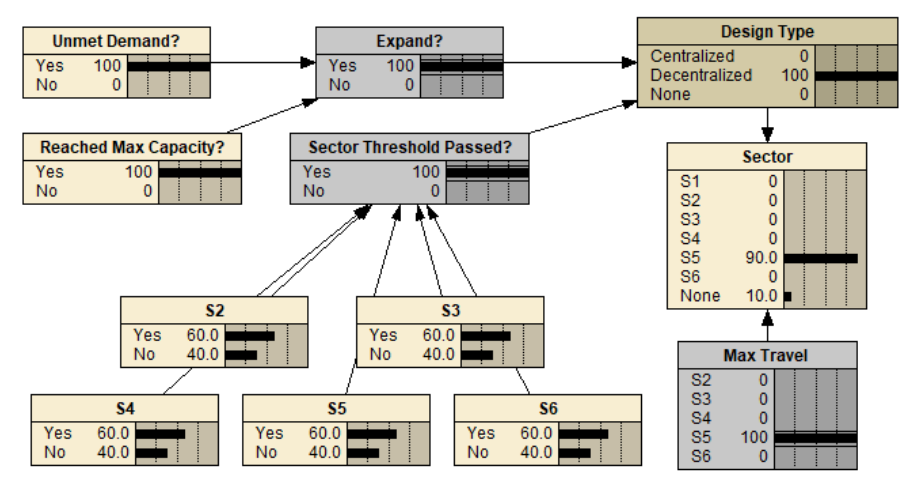

\section{FIGURE 5 - BAYESIAN NETWORK DECISION RULES WITH OBSERVATIONS}

A powerful property of the $\mathrm{BN}$ is that inference can be used to understand both cause-to-effect, as above, as well as be used to investigate effect-to-cause. That is, in the above example, observations were entered to understand in which sector to expand. Going the other way, the decision maker may want to investigate what conditions are necessary for a centralized design. This is illustrated in the following figure where centralized in the "Design Type" node has been observed.

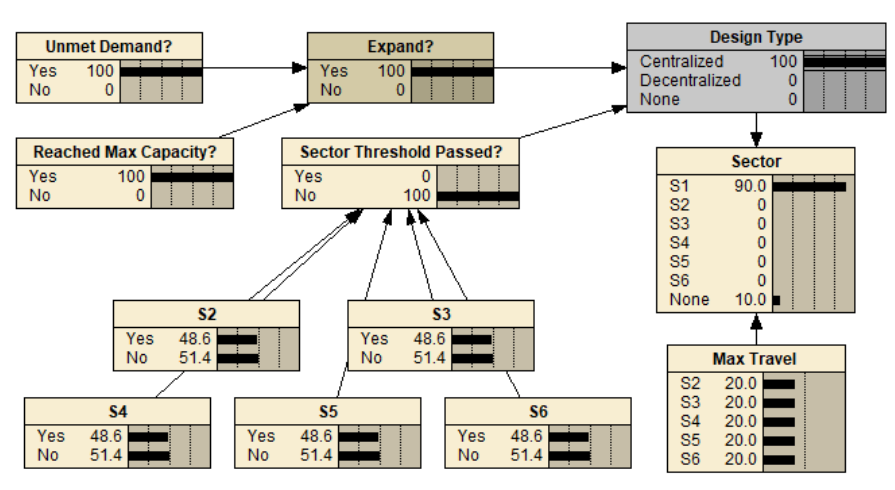

\section{FIGURE 6 - BACKWARDS INFERENCE WITH BAYESIAN NETWORK}

The necessary conditions for centralized design are therefore: the system has to have unmet demand, maximum capacity has to have been reached and the decentralized sectors have not passed the capacity thresholds. Furthermore, the sector recommended for expansion is $\mathrm{S} 1$ which represents the central site. The BN was tested against previous work [18, 23] to ensure similar results and functioning. The simulation can now be ran similarly to Step 1 and Step 2 but with the revenues and costs reflecting whether the sectors have been upgraded as decided by the BN.

\subsection{Step 4: Design Space Evaluation}

With the model and decision rules defined, the full design space can be evaluated to find the optimal designs with flexible strategies. The model, BN and decision rules were executed in MATLAB with the design space explored using a Monte Carlo approach and simulated annealing for optimization. Similar to Step 1, the initial capacity was optimized to understand the initial robust margins of the system. However, the system can now also execute decision rules decided by the $\mathrm{BN}$ for expansion as defined in Step 3. The optimal design is summarized in the following table.

\section{TABLE 3 - ENPV OF FLEXIBLE DECENTRALIZED DESIGN UNDER UNCERTAINTY}

\begin{tabular}{|c|c|c|c|c|c|c|c|}
\hline \multirow{2}{*}{$\begin{array}{c}\text { ENPV } \\
\text { (S\$ }\end{array}$} & \multicolumn{6}{|c|}{ Initial Capacity } & \multirow{3}{*}{$\begin{array}{c}\text { Total } \\
\text { Capacity } \\
\text { (tpd) }\end{array}$} \\
\hline & Sector & Sector & Sector & Sector & Sector & Sector & \\
\hline Million) & 1 & 2 & 3 & 4 & 5 & 6 & \\
\hline 254 & 1400 & 900 & 850 & 800 & 550 & 500 & 5000 \\
\hline
\end{tabular}

It can be seen that the ENPV of the flexible decentralized design is higher than the fixed decentralized design of S\$250 million and fixed centralized design of $\mathrm{S} \$ 251$ million. The total initial capacity of the flexible design is lower than the fixed design which may indicate that it is better to defer initial capacity investment to allow for later expansion. The value of flexibility is calculated by

$$
\begin{aligned}
V O F & =E N P V_{\text {Flexible }}-E N P V_{\text {Benchmark }} \\
& =254-250=S \$ 4 \text { million }
\end{aligned}
$$




\subsection{Step 5: Resilience Analysis}

The key study of this paper is to understand how robust and flexible strategies can be used in order to maximize the system lifecycle value and thus make the system resilient to future uncertainties. This step varies the volatility of the stochastic demand in order to assess the effects on the ENPV of the system. Figure 7 illustrates the effect of varying the volatility on the total domestic solid waste projection. It is shown that there is a wider "spread" of demands with increased volatility.

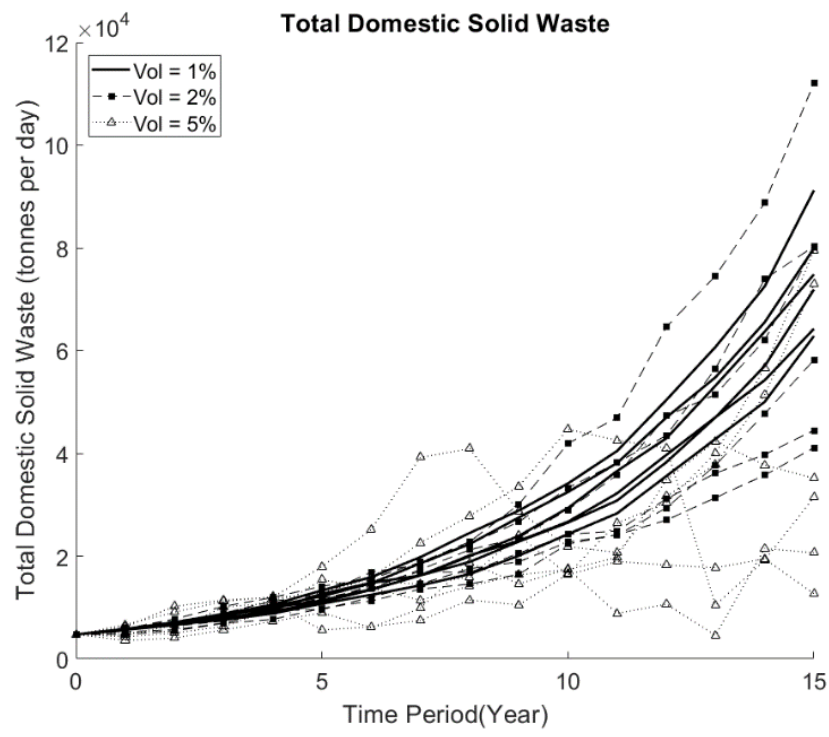

FIGURE 7 - TOTAL DOMESTIC SOLID WASTE PROJECTIONS

The following analyses were based on the WTE system with optimized configurations as found in Step 4 unless stated otherwise.

\subsubsection{Effect of Volatility}

When varying the volatility through the simulations, it can be seen that, although the total number of upgrades in the sectors are similar, the distribution of sectors that are upgraded are different. This is shown in Figure 8. Sector 1, the centralized site, has the most number of expansions over 2000 simulations due to the lowest transportation cost in that sector. The distribution over the other five sectors follow the distribution of transportation costs similarly. With increasing volatility, the system allocates more upgrades to $\mathrm{S} 1$ which is the centralized site. This is due to the condition in the $\mathrm{BN}$ that all decentralized sectors need to exceed the threshold before decentralized expansion takes place. With increased volatility, there are large fluctuations in demand which causes the $\mathrm{BN}$ to select an expansion but not every decentralized sector may simultaneously have the sufficient spike in demand to warrant decentralized expansion. In the simulations with lower volatility, the demand increases steadily such that the condition that all the sector exceeds the threshold happen at similar times.

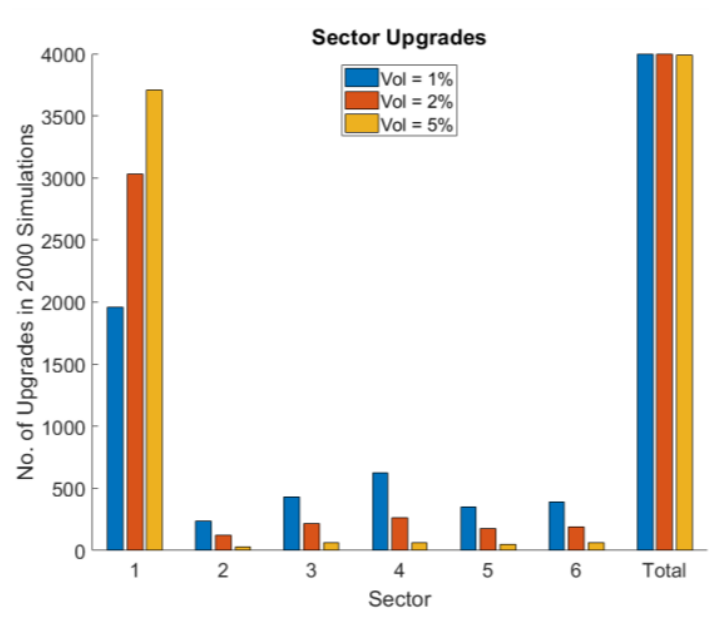

FIGURE 8 - SECTOR UPGRADES

The increased volatility also slightly reduces the total number of expansions and therefore the average number of expansions is also reduced with increasing volatility as shown in Table 4. This further means that the average number of years between expansions is longer due to the reduced number of expansions. This may be explained by noting that in the simulations, only volatility was varied and drift, the upwards trend, was held constant which means that spikes of increased demand could also be followed by a dip in demand. Without a steady increase in demand, fewer simulations reached the expansion threshold and therefore a lower number of expansions occurred.

\section{TABLE 4 - EFFECT OF VOLATILITY}

\begin{tabular}{c|c|c}
\hline Volatility & $\begin{array}{c}\text { Average No. } \\
\text { Expansions }\end{array}$ & $\begin{array}{c}\text { Average No. Years } \\
\text { between Expansions }\end{array}$ \\
\hline $1 \%$ & 2 & 1 \\
$2 \%$ & 2 & 1.0055 \\
$5 \%$ & 1.9935 & 1.1680
\end{tabular}

The years in which there were expansions are shown in Figure 9. The set of solid lines indicate a volatility of $1 \%$, the square markers have a volatility of $2 \%$ and empty circles have a volatility of $5 \%$.

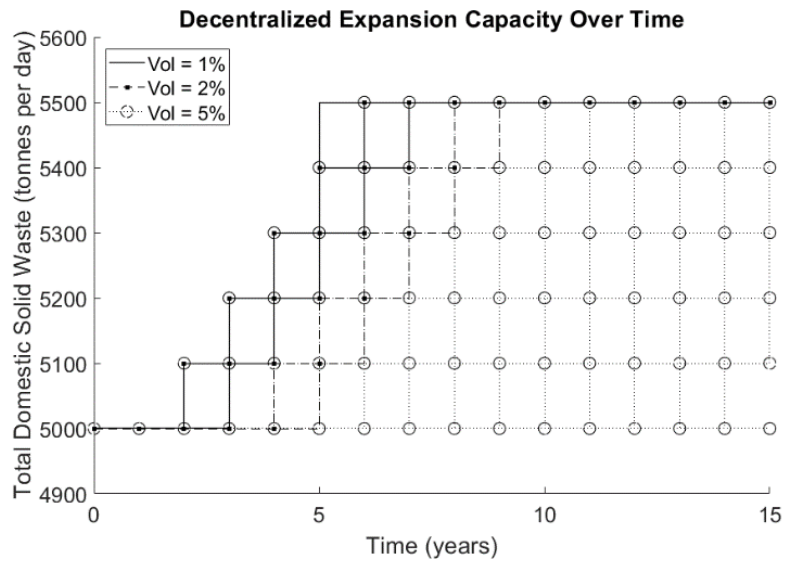

FIGURE 9 - DECENTRALIZED EXPANSION CAPACITY OVER TIME 
It is seen that for low volatility, expansion occurs early on with a smaller spread of years in which expansion took place. As the volatility increases, the distribution of years in which expansion took place also increases. This is shown by the empty circle marker in more points of expansion.

\subsubsection{Effect of Decentralization}

The model also incorporated a decentralization threshold which controls whether the expansion will occur in a decentralized or centralized manner. By setting this threshold high, the expansion only happens in a centralized manner. The effect of volatility and decentralization are shown in Figure 10. It can be seen that for both volatility plots, the lowest ENPV is with centralized expansion (left-most line) and the ENPV improves with decentralization. The effect of volatility decreases the gradient of the cumulative probability plot due to the increased spread of demand projections and thus ENPVs. By comparing these results, it is shown to be beneficial in adopting the decentralized design

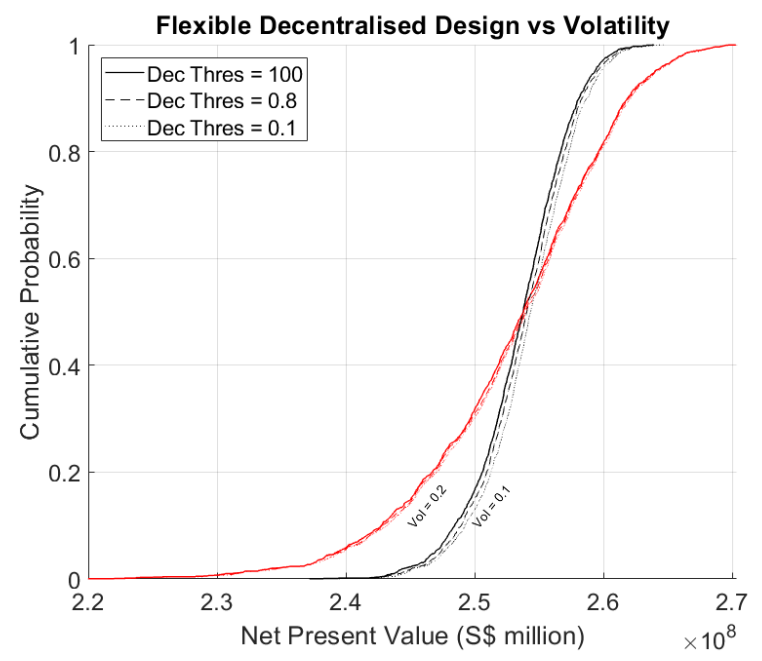

FIGURE 10 - ENPV VS VOLATILITY

\subsubsection{Effect of Robust and Flexible Strategies}

The simulation is now ran for a range of initial capacities and average number of expansions to investigate the effect of robustness and flexibility. The maximum capacity of the system was increased to give a larger range of ENPVs and results. The average number of expansions over 2000 simulations is taken as a proxy for flexibility and varied by changing the number of simultaneous unit expansions at a time. That is, the larger the step increase in the capacity during each upgrade, the fewer total upgrades are implemented and hence the lower average number of expansions. The result of this with volatility $=1 \%$ is shown in Figure 11. It is shown that by having a low initial capacity and meeting the demand on the system through a high number of expansions yields a low ENPV. This is due to each expansion incurring a cost which negates the benefit in allowing for the flexibility. Similarly, the value of flexibility is also negated where there is a high initial capacity and a high average number of expansions. In this system, the highest ENPVs are obtained with a large initial capacity and fewer number of expansions. This could be due to having all demand captured, thus generating more revenue, with no limitations on capacity. However, consistent with earlier experiments, having some flexibility is better than none, as shown by the slight peak where there are two or three expansions. The simulations with no increase in capacity over time are unable to take advantage of increased demand over time and therefore gives a lower ENPV.

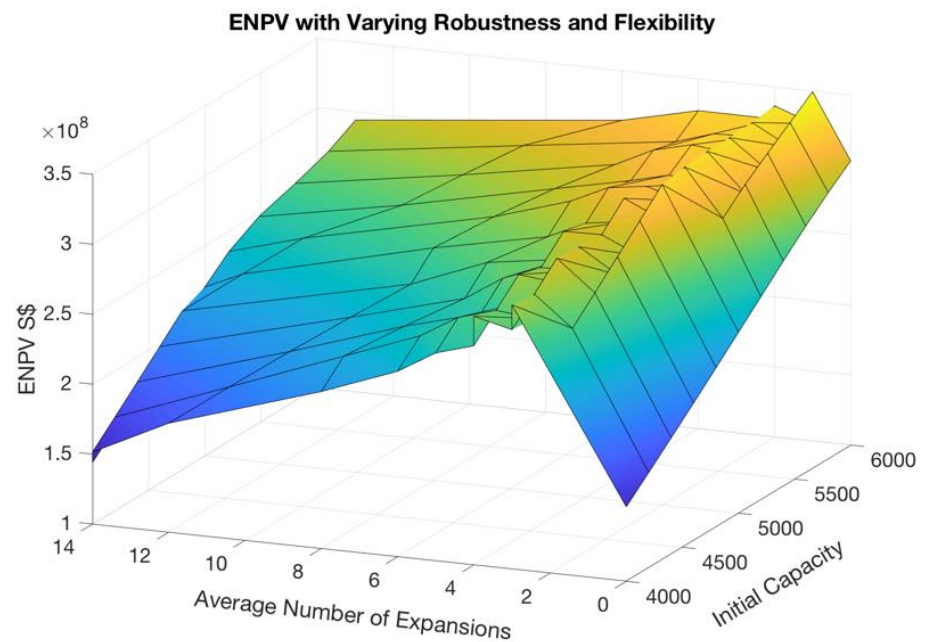

\section{FIGURE 11 - EFFECT OF ROBUSTNESS AND FLEXIBILITY ON ENPV $(\mathrm{VOL}=1 \%)$}

For this WTE system, it may be seen that the best strategy is where there is a high initial capacity, such that the maximum capacity takes some time to be reached, and two or three expansions. The possible configurations are summarized in Figure 12.

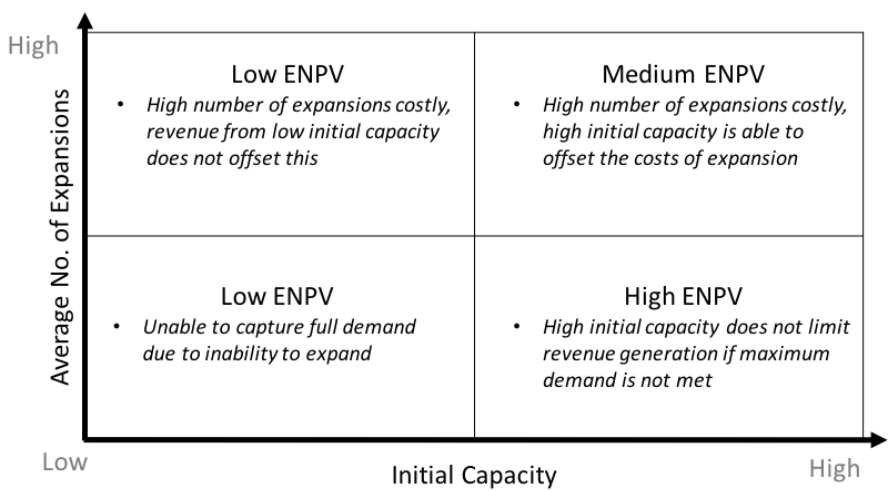

\section{FIGURE 12 - SUMMARY OF ROBUST AND FLEXIBLE STRATEGIES}

The effects of volatility on these parameters are also considered. The variation of initial capacity and volatility are show in Figure 13. Although for all volatilities, there is 
increasing ENPV with increasing initial capacity, as the volatility increases, the ENPV decreases. This is due to the higher initial capacities being able to capture demand without being restricted. The higher volatilities, however, may be more prone to exceeding the maximum capacities, resulting in reduced ENPV.

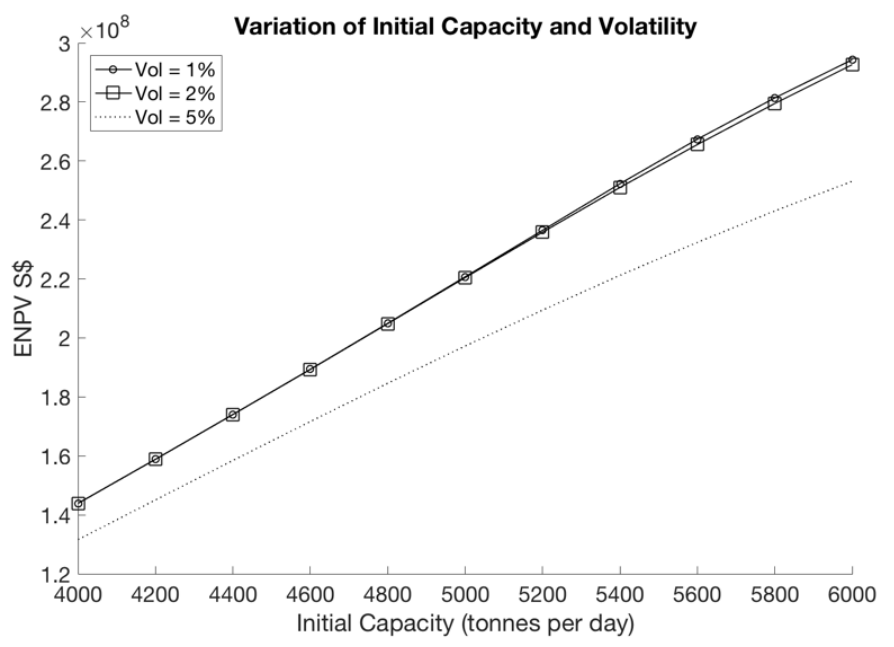

\section{FIGURE 13 - VARIATION OF INITIAL CAPACITY WITH VOLATILITY}

The average number of expansions can also be seen to follow a similar trend in Figure 14 where the higher volatility results in a lower ENPV. The peak showing the optimum number of expansions is clearly shown here and occurs around three expansions regardless of volatility.

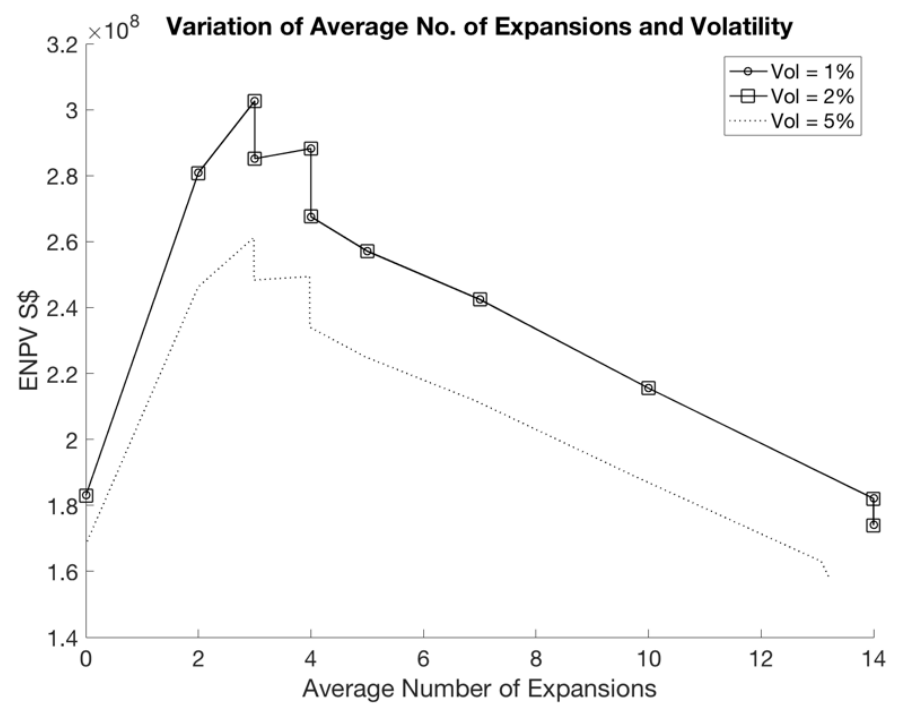

FIGURE 14 - VARIATION OF AVERAGE NO. OF EXPANSIONS WITH VOLATILITY

\section{CONCLUSION AND FURTHER WORK}

This study presents a framework to design resilient engineering infrastructure systems through robust and flexible strategies such that the system lifecycle value, as measured by NPV, is maximized. This was applied to a WTE system in Singapore where the robust strategy involved optimizing for initial capacity and flexibility was implemented through the use of Bayesian Networks to select and execute decentralized expansion sites. The Bayesian Network shows promise, giving similar results to previous studies, and should be considered further where decisions need to be more complex, perhaps involving qualitative and quantitative data. The results show that a decentralized design performed better than a centralized design and increasing volatility generally made the system perform worse since not all demand could be converted to revenue. Flexibility shows increased value compared to a fixed design. However, when the system is allowed to upgrade too often, the costs of implementation negates the revenue increase. The better design is to have a high initial capacity, such that there is less restriction on the demand with two or three expansions.

Future work could include making the Bayesian Network more complex to capture more types of uncertainty in the decision making process. Shocks can also be introduced into the system. Currently the demand is assumed to be of a constant volatility and drift. However, this is rarely the case over a prolonged timespan and sudden changes in demand could be simulated.

\section{REFERENCES}

[1] Holling, Crawford S. "Resilience and stability of ecological systems." Annual review of ecology and systematics, Vol. 4.1 (1973): pp. 1-23.

[2] Sheffi, Yossi, and James B. Rice Jr. "A supply chain view of the resilient enterprise." MIT Sloan management review Vol. 47.1 (2005): p 41.

[3] McManus, Sonia, et al. "Resilience management: a framework for assessing and improving the resilience of organisations." (2007).

[4] Rutter, Michael. "Psychosocial resilience and protective mechanisms." American journal of orthopsychiatry 57.3 (1987): p. 316.

[5] Hollnagel, Erik, David D. Woods, and Nancy Leveson. Resilience engineering: Concepts and precepts. Ashgate Publishing, Ltd., (2007).

[6] Hamel, Gary, and Lisa Valikangas. "The quest for resilience." Harvard business review, Vol. 81.9 (2003) pp.52-65.

[7] Mak, W. H. Jonathan and Clarkson, P. John. "Towards the Design of Resilient Large-scale Engineering Systems." Procedia CIRP Vol. 60 (2017): pp 536-541, DOI: https://doi.org/10.1016/j.procir.2017.01.034.

[8] Fricke E, Schulz AP. "Design for changeability (DfC): Principles to enable changes in systems throughout their entire lifecycle". Systems Engineering, Vol. 8(4), (2005). pp. 342-359.

[9] Woods, D.D., "Essential Characteristics of Resilience". In Resilience engineering: Concepts and precepts. 2006. pp. $21-$ 34. 
[10] Ross AM. "Managing Unarticulated Value: Changeability in Multi-Attribute Tradespace Exploration". Massachusetts Institute of Technology. (2006).

[11] Gessner GA, Jardim J. "Bridge Within a Bridge". Civil Engineering Vol. 68(10), (1998), pp.44-47.

[12] Filippini, Roberto, and Andrés Silva. "A modeling framework for the resilience analysis of networked systemsof-systems based on functional dependencies." Reliability Engineering \& System Safety Vol. 125 (2014): pp 82-91.

[13] Vugrin, Eric D., Drake E. Warren, and Mark A. Ehlen. "A resilience assessment framework for infrastructure and economic systems: Quantitative and qualitative resilience analysis of petrochemical supply chains to a hurricane." Process Safety Progress Vol. 30.3 (2011): pp 280-290.

[14] Hosseini, Seyedmohsen, and Kash Barker. "Modeling infrastructure resilience using Bayesian networks: A case study of inland waterway ports." Computers \& Industrial Engineering Vol. 93 (2016): pp 252-266.

[15] Ouyang, Min, and Zhenghua Wang. "Resilience assessment of interdependent infrastructure systems: With a focus on joint restoration modeling and analysis." Reliability Engineering \& System Safety Vol. 141 (2015): pp 74-82.

[16] Hosseini, Seyedmohsen, Kash Barker, and Jose E. RamirezMarquez. "A review of definitions and measures of system resilience." Reliability Engineering \& System Safety Vol. 145 (2016):pp 47-61.

[17] Cardin, Michel-Alexandre. "Enabling flexibility in engineering systems: a taxonomy of procedures and a design framework." Journal of Mechanical Design Vol. 136.1 (2014): p 011005.

[18] Liu, Ziqi, "Determining the Optimal Configuration of a Mechanical Biological Treatment System under Uncertainty." Master's Thesis. National University of Singapore, Singapore. (2017).

[19] Trigeorgis, Lenos. "Real options: Managerial flexibility and strategy in resource allocation”. MIT press, (1996).

[20] Cardin, Michel-Alexandre, et al. "Empirical evaluation of procedures to generate flexibility in engineering systems and improve lifecycle performance." Research in Engineering Design Vol. 24.3 (2013): pp 277-295.

[21] Mikaelian, Tsoline. "An integrated real options framework for model-based identification and valuation of options under uncertainty". PhD Thesis. Massachusetts Institute of Technology, USA. (2009).

[22] $\mathrm{Hu}$, Junfei, and Cardin, Michel-Alexandre. "Generating flexibility in the design of engineering systems to enable better sustainability and lifecycle performance." Research in Engineering Design Vol. 26.2 (2015): pp 121-143.

[23] Cardin, Michel-Alexandre, and Junfei Hu. "Analyzing the tradeoffs between economies of scale, time-value of money, and flexibility in design under uncertainty: Study of centralized versus decentralized waste-to-energy systems." Journal of Mechanical Design Vol. 138.1 (2016): p 011401.

[24] Data Science Division. Solid Waste Management - Total Domestic Waste Disposed. Government of Singapore. (2016) Retrieved from: http://data.gov.sg/dataset?q=Solid+Waste
[25] Pearl, Judea. Probabilistic reasoning in intelligent systems: networks of plausible inference. Elsevier (1988)

[26] Nielsen, Thomas Dyhre, and Finn Verner Jensen. Bayesian Networks and Decision Graphs. Springer Science \& Business Media (2009).

[27] Netica. (2018) Retrieved from: https://www.norsys.com/ 